\title{
Measurement of Total and Compartmental Lung Volume Changes in Newborns by Optoelectronic Plethysmography
}

\author{
RAFFAELE L. DELLACA', MARIA L. VENTURA, EMANUELA ZANNIN, MIRIA NATILE, ANTONIO PEDOTTI, \\ AND PAOLO TAGLIABUE
}

\begin{abstract}
Dipartimento di Bioingegneria [R.L.D., E.Z., A.P.], Politecnico di Milano, Milano 20133, Italy; Neonatology and Neonatal Intensive Care
\end{abstract} Unit [M.L.V., M.N., P.T.], S. Gerardo Hospital, Monza 20052, Italy

\begin{abstract}
The study of respiratory mechanics in infants requires a noninvasive accurate measurement of the lung volume changes $\left(\Delta V_{\mathrm{L}}\right)$. Optoelectronic plethysmography (OEP) allows the assessment of $\Delta V_{\mathrm{L}}$ through the measurement of the chest wall surface motion and it has been proved to be accurate in adults. The aim of this study was to apply OEP to newborns and to validate it by comparison to pneumotachography. Twenty term and preterm newborns $(\mathrm{GA}=34 \pm 5 \mathrm{wk})$ in stable condition were studied during 1 to 2 min periods of quiet breathing in supine position. Airway opening flow was measured by applying a facemask connected to a pneumotachograph (PNT) and integrated to provide the $\Delta V_{\mathrm{L}}$. Chest wall volume changes were simultaneously measured by OEP. The tidal volume values measured by pneumotachography and by OEP were compared for each breath. A total of 771 breaths from all patients were considered. Bland-Altmann analysis showed a mean difference of $-0.08 \mathrm{~mL}$ and a limit of agreement ranging from -2.98 to $2.83 \mathrm{~mL}$. Linear regression analysis demonstrated good correlation between the two techniques $\left(r^{2}=0.95, q=1.00 \mathrm{~mL}, m=0.96\right)$. OEP provides accurate measurements of $\Delta V_{\mathrm{L}}$ in newborns and may be useful to study respiratory mechanics and breathing patterns during spontaneous breathing and mechanical ventilation. (Pediatr Res 67: 11-16, 2010)
\end{abstract}

$\mathrm{T}$

The study of respiratory mechanics and control of breathing ideally requires an accurate and noninvasive measurement of lung volume changes $\left(\Delta V_{\mathrm{L}}\right)$ not interfering with patients' spontaneous activity. Unfortunately, the currently available methods for the measurement of $\Delta V_{\mathrm{L}}$ in infants present several intrinsic and unresolved limitations.

Most of the techniques require either connections to the airway opening or critical patient- and posture-dependent calibration procedures. Connecting to the airway opening may introduce leaks, annoy the infant, and affect the breathing pattern $(1,2)$, while the use of patient-dependent calibration procedures can lead to inaccurate measurements when a pa-

Received March 16, 2009; accepted September 6, 2009

Correspondence: Raffaele L. Dellaca', Ph.D., Dipartimento di Bioingegneria, Politecnico di Milano, Piazza Leonardo da Vinci 32, I-20133 Milano, Italy; e-mail: raffaele.dellaca@polimi.it

Supported, in part, by the Istituto Italiano Tecnologico (IIT), Politecnico di Milano Unit.

Supplemental digital content is available for this article. Direct URL citations appear in the printed text and are provided in the HTML and PDF versions of this article on the journal's Web site (www.pedresearch.org). tient's condition or posture changes with respect to the baseline state (3). Moreover, none of the existing techniques have proved to be reliable in tracking long-term changes in endexpiratory lung volume (EELV) due to integration drift or problems in the measurements stability $(4,5)$.

Optoelectronic plethysmography (OEP) is a noninvasive technique, which estimates chest wall volume $\left(V_{\mathrm{CW}}\right)$ by measuring the three-dimensional position of several reflective markers placed on the patient's thorax $(6-8)$. This technique has proved to be reliable in adults in different postures and conditions $(7,8)$.

OEP does not require a connection to the airway opening or subject-specific calibration procedures. It provides an accurate measurement of the $V_{\mathrm{CW}}$ and it allows, through the use of subsets of the markers placed on the thorax, the measurement of separate compartments, such as the rib cage, abdomen, or smaller components (9). Being based on the direct measurement of chest wall volumes, OEP is not affected by integration drift and can be used to track breath-by-breath changes of EELV (10).

The aim of this study was to develop an appropriate experimental set-up and a geometrical model to apply OEP to term and preterm newborns and to validate the system by comparison with pneumotachography.

\section{METHODS}

All measurements were performed in a tertiary level NICU. The study was approved by the institutional ethical committee of the San Gerardo Hospital (Monza, Italy), and informed consent was obtained from parents.

Study population. Any infant in a stable condition at the time of the measurement and with no lung dysfunction was eligible for the study.

Experimental protocol. Infants were studied during quiet breathing laying in supine position on an infant warmer (Air-Shields Vicker, Hatboro, PA). Airflow was measured at the mouth using a facemask connected to a pneumotachograph (PNT). To avoid leaks, the mask was held firmly in place by an operator during data recording for $2 \mathrm{~min}$ at maximum.

Abbreviations: $\mathbf{A B} \%$, percentage contribution of the abdomen to $V_{\mathrm{T}, \mathrm{CW}}$; $\%$ diff, percentage difference between $V_{\mathrm{T}, \mathrm{L}}$ and $V_{\mathrm{T}, \mathrm{CW}} ; \boldsymbol{\Delta} \boldsymbol{V}_{\mathrm{L}}$, lung volume changes; EELV, end-expiratory lung volume; $\boldsymbol{\theta}$, phase angle; PNT, pneumotachograph; OEP, optoelectronic plethysmography; RIP, respiratory inductive plethysmography; $\boldsymbol{V}_{\mathbf{C W}}$, chest wall volume; $\boldsymbol{V}_{\mathbf{T}, \mathbf{L}}$, tidal volume computed from $\Delta V_{\mathrm{L}} ; \boldsymbol{V}_{\mathbf{T}, \mathbf{C W}}$, chest wall tidal volume 
Measurements. Detailed descriptions of the OEP for the analysis of the $V_{\mathrm{CW}}$ in standing, seated, and supine adults have been published previously $(8,11,12)$.

To apply OEP in supine newborns, 24 hemispheric reflective markers (6 $\mathrm{mm}$ diameter) were positioned on the anterior thoracicabdominal surface from the clavicles to the anterior superior iliac spines at the intersection between predefined vertical and horizontal lines (Fig. 1).

Six horizontal lines were designated for placing the markers: 1) the clavicular line; 2) the nipples; 3) the xiphoid process; 4) the lower costal margin; 5) umbilicus; 6) superior iliac spine; as well as five vertical lines: 1) the midline (along the sternum and continuing caudally below the xiphoid through the umbilicus); 2-3) two parasternal lines; 4-5) two midaxillary lines. To better outline the costal margin, two markers were added between the parasternal and the midaxillary lines. Similarly, to increase marker density in the abdomen, one more marker was added between the xiphoid process and the umbilicus.

The markers were placed on the skin using bi-adhesive hypoallergenic tape. The three-dimensional position of each marker was measured by an automatic optoelectronic motion analyzer (OEP System, BTS, Milan, Italy) at a frequency rate of $60 \mathrm{~Hz}$ using six specially designed infrared video cameras provided with infrared flashing light-emitting diodes. Cameras were positioned so that each marker was simultaneously seen by at least two cameras (Fig. 2) to reconstruct their three-dimensional position and displacement during respiration by stereo-photogrammetric methods. Three cameras were placed on one lateral side about $1 \mathrm{~m}$ from the bed. Three additional ones were placed symmetrically on the other side of the bed. The four cameras on the corners were placed about $70 \mathrm{~cm}$ above the subject while the two in the middle were placed $1.1 \mathrm{~m}$ above the subject; all of them were slanted downward. The operative calibrated volume was large enough to include the whole trunk of the infant lying on the bed.

Total $V_{\mathrm{CW}}$ was finally determined by approximating chest wall surface by 34 triangles connecting the markers and computing the volume enclosed by all these triangles. The posterior surface of the chest wall (hidden in supine position) was defined by nine "virtual" markers assumed to lie on the supporting plane. The supporting plane was assumed to be parallel to the plane of the bed and $5 \mathrm{~mm}$ below the most posterior lateral marker (lateral markers being: 7, 8, 12, 15, 20, and 24, see Fig. 1). The positions of the virtual markers were determined by projecting onto the reference plane the initial frontal coordinates (from the first image frame) of the following points: 1) the average between marker 4 and $7 ; 2$ ) the average between marker 6 and $8 ; 3$ ) marker 11;4) marker 14;5) the average between marker 20 and 21;6) marker $21 ; 7)$ marker 22;8) marker 23; and 9) the average between marker 23 and 24.

To compute $V_{\mathrm{CW}}$ and its division in compartments, such as rib cage $\left(V_{\mathrm{RC}}\right)$ and abdomen $\left(V_{\mathrm{AB}}\right)$, all the considered triangles must define a closed surface surrounding the compartment. This condition is satisfied only if the position of the markers does not differ significantly from the indications of Figure 1. If a marker were placed incorrectly, the previous mathematical procedure (8)

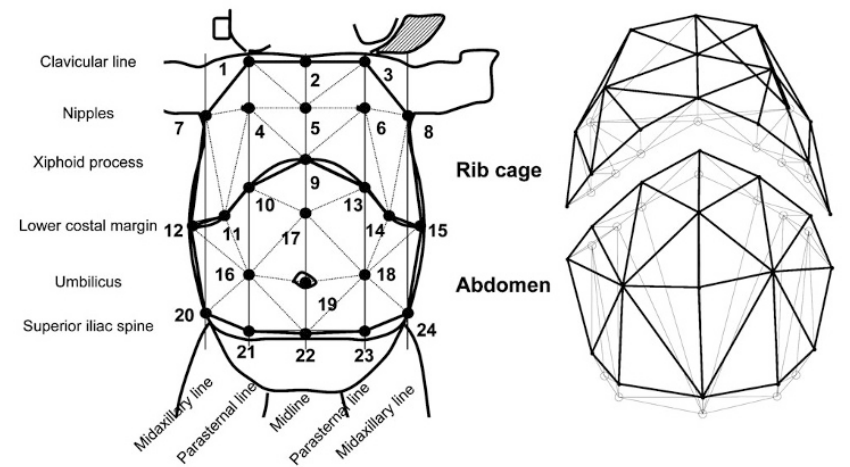

Figure 1. Markers positioning and geometrical model for OEP measurement in newborns. Left panel: Markers' positioning and chest wall compartments for optical analysis in newborns in supine position. Transverse lines: clavicular line; manubriosternal joint, lower costal margin, and anterior superior iliac crest. Craniocaudal lines: midsternal line (extended to the abdomen), the midpoints of the interval between the midsternal and the midaxillary lines; and two midaxillary lines. Right panel: Three-dimensional model of the chest wall from one representative model. Black thick lines: triangles connecting real markers (solid circles); gray lines: triangles connecting to at least one "virtual" marker (open circles). The two compartments are drawn separately by introducing an offset on the craniocaudal direction for clarity.

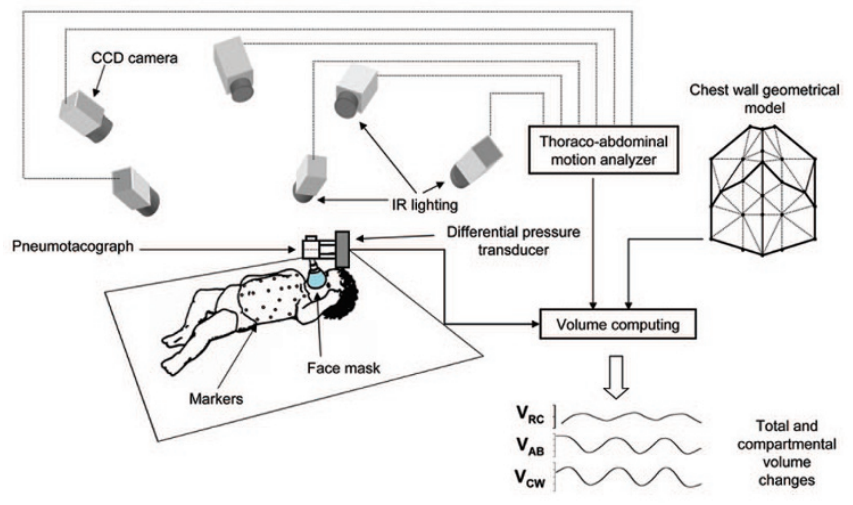

Figure 2. Experimental setup for the application of optoelectronic plethysmography in newborns. See methods section for details.

would provide erroneous results with no warnings to the user. Here, we developed a new algorithm, which is capable of automatically identifying improperly positioned markers (see supplementary material http:// links.lww.com/PDR/A54). When markers are properly positioned, the two algorithms provide exactly the same volumes.

Measurements of lung volume changes. The subjects breathed through a facemask connected to a heated mesh-type (PNT) (Hans-Rudolph 3700A) coupled with a differential pressure transducer (Pascaline PXLA02X5DN) for the measurement of airway opening flow $\left(\dot{V}_{\mathrm{AO}}\right)$. The flow signal was sampled at $960 \mathrm{~Hz}$. Corrections for humidity, pressure, and temperature changes of the gas between inspiration and expiration were not applied. Lung volume changes were obtained by numerical integration of the flow signal. The resulting volume drift was corrected by estimating the linear trend of the volume trace over time by linear regression analysis and by subtracting it from the integrated signal, providing a drift-corrected $\Delta V_{\mathrm{L}}$ trace.

Data analysis. For each infant data were recorded during $\sim 1$ to $2 \mathrm{~min}$ of quiet breathing. All the recorded breaths not corrupted by clearly identifiable artifacts (such as disconnection of the mask, presence of leaks, or major patient movements) were manually selected. For each breath, end-expiratory and end-inspiratory points were identified in the $\Delta V_{\mathrm{L}}$ trace. These points were used to compute the tidal volume of each breath from both $\Delta V_{\mathrm{L}}$ and $V_{\mathrm{CW}}$ tracings ( $V_{\mathrm{T}, \mathrm{L}}$ and $V_{\mathrm{T}, \mathrm{CW}}$, respectively).

The Bland-Altman analysis, which is a plot of the difference between data measured by two methods against their mean, was performed on raw data to test the agreement of the two ways of measuring $\Delta V_{\mathrm{L}}$ (13). The same data were also used to perform a linear regression analysis between the techniques.

OEP is based on the approximation of the chest wall shape and dimensions by a group of triangles. If this approximation were not accurate, the accuracy of the measurements could be affected and biased by the chest wall shape, size, or motion pattern. To evaluate this issue, body weight and GA were also added as independent variables in the regression analysis.

The percentage difference (\%diff) between OEP and PNT was computed for each single breath as follows:

$$
\% \text { diff }=\frac{\left(V_{\mathrm{T}, \mathrm{L}}-V_{\mathrm{T}, \mathrm{CW}}\right)}{\Delta V_{\mathrm{T}, \mathrm{L}}} \times 100
$$

The percentage differences were then averaged for each patient.

There are no available gold standards for the measurement of compartmental volumes in newborns. Even if the absolute compartmental accuracy cannot be evaluated, it is still possible to detect differences in the accuracy of the rib cage or the abdominal compartment resulting from inappropriate distribution of markers on the chest wall by studying the relationship between $\%$ diff and the percentage contribution of each compartment. If the rib cage and the abdominal volumes were measured with different accuracies, the most inaccurate would negatively affect the overall accuracy depending on how much it contributes to total $V_{\mathrm{T}} \mathrm{CW}$, introducing a trend in this relationship.

To quantify thoracoabdominal asynchronies, the following parameters were calculated from the total and compartmental $V_{\mathrm{CW}}$ tracings: rib cage and abdominal contribution to $V_{\mathrm{T}, \mathrm{CW}}(\mathrm{RC} \%$ and $\mathrm{AB} \%)$ and phase angle between abdominal and thoracic motions $(\theta)$. The phase angle was calculated, as previously suggested (14), through the analysis of Lissajous figures obtained by plotting rib cage versus abdominal volume tracings (Konno-Mead plots). When rib cage and abdomen are synchronous, a narrow or closed loop is produced, which widens with increasing asynchrony. The width of the loop is a measure of asynchrony and can be quantified by the phase angle $(\theta)$, which 
is $\theta=\sin ^{-1}(\mathrm{~m} / \mathrm{s})$ for $\theta<90^{\circ}$, and $\theta=180^{\circ}-\sin ^{-1}(\mathrm{~m} / \mathrm{s})$ for $\theta>90^{\circ}$, where $m$ is the width of Lissajous figure at mid-rib cage excursion, and $s$ is the width of Lissajous figure at its largest abdominal excursion. Phase angle can range from $0^{\circ}$ (synchronous) to $180^{\circ}$ (paradoxical breathing). Whether outward motion of the abdomen preceded or lagged the motion of the rib cage was determined by considering the direction of Lissajous figures (anticlockwise or clockwise). Negative angles were used for anticlockwise direction, indicating an outward motion of the abdomen preceding the rib cage.

The overall accuracy of OEP in measuring tidal volume was evaluated by Bland-Altman and linear regression analysis pooling all breaths from all patients. Multiple linear regression analysis between $V_{\mathrm{T}, \mathrm{L}}$ and $V_{\mathrm{T}, \mathrm{CW}}$ on pooled data including body weight and GA as independent variable was performed to examine the extent to which they alter the regression coefficient. Data from each patient, expressed as mean $\pm \mathrm{SD}$, were also reported.

\section{RESULTS}

A total of 20 newborns with body weight ranging between 1300 and $3790 \mathrm{~g}$ at the time of the study were recruited. Relevant data on the patient population are reported in Table 1.

A representative experimental tracing with total and compartmental chest wall volumes, $\dot{V}_{\mathrm{AO}}$, and $\Delta V_{\mathrm{L}}$ is shown in Figure 3. Rib cage volume fluctuates around $350 \mathrm{~mL}$, abdominal volume around $510 \mathrm{~mL}$, and $V_{\mathrm{CW}}$ around $860 \mathrm{~mL}$. These values represent the whole volume of the rib cage, abdomen, and chest wall, and they are not related to lung volume. However, changes in $V_{\mathrm{CW}}$ can be associated to lung volume changes and compared with data obtained by PNT. Altogether, a total of 771 breaths were selected, providing 1542 volume changes. The Bland-Altman analysis (Fig. 4) showed a good agreement between $V_{\mathrm{T}, \mathrm{L}}$ and $V_{\mathrm{T}, \mathrm{CW}}$. The mean difference was $-0.08 \mathrm{~mL}$ indicating that, when considering the whole patients population, there is no significant bias in the measurement. The limit of agreement ranged from -2.98 to $2.83 \mathrm{~mL}$, and we did not find any relationship between the difference and the mean of the measurements, suggesting that OEP does not introduce any systematic error related to the

Table 1. Patients' characteristics

\begin{tabular}{llcccc}
\hline $\begin{array}{c}\text { Subject } \\
\text { no. }\end{array}$ & Sex & $\begin{array}{c}\text { GA } \\
(\mathrm{wk})\end{array}$ & $\begin{array}{c}\text { PMA } \\
(\mathrm{wk})\end{array}$ & $\begin{array}{c}\text { BW } \\
(\mathrm{g})\end{array}$ & $\begin{array}{c}\text { Weight at } \\
\text { test }(\mathrm{g})\end{array}$ \\
\hline 1 & M & 39 & 39 & 3570 & 3360 \\
2 & $\mathrm{~F}$ & 38 & 38 & 3500 & 3400 \\
3 & $\mathrm{M}$ & 39 & 39 & 3500 & 3400 \\
4 & $\mathrm{~F}$ & 33 & 33 & 2500 & 2200 \\
5 & $\mathrm{~F}$ & 40 & 40 & 3500 & 3300 \\
6 & $\mathrm{M}$ & 39 & 39 & 3200 & 2900 \\
7 & $\mathrm{M}$ & 31 & 35 & 1700 & 2000 \\
8 & $\mathrm{M}$ & 27 & 36 & 700 & 1800 \\
9 & $\mathrm{~F}$ & 41 & 41 & 3300 & 3200 \\
10 & $\mathrm{M}$ & 39 & 39 & 2800 & 2600 \\
11 & $\mathrm{M}$ & 29 & 33 & 1110 & 1300 \\
12 & $\mathrm{M}$ & 38 & 44 & 3100 & 3790 \\
13 & $\mathrm{~F}$ & 29 & 31 & 1730 & 1520 \\
14 & $\mathrm{~F}$ & 32 & 34 & 1900 & 1940 \\
15 & $\mathrm{~F}$ & 39 & 39 & 3890 & 3360 \\
16 & $\mathrm{M}$ & 30 & 32 & 1390 & 1510 \\
17 & $\mathrm{~F}$ & 30 & 30 & 1320 & 1560 \\
18 & $\mathrm{M}$ & 30 & 30 & 1415 & 1690 \\
19 & $\mathrm{~F}$ & 29 & 34 & 1340 & 1720 \\
20 & $\mathrm{~F}$ & 29 & 34 & 1190 & 1640 \\
Mean \pm SD & - & $34 \pm 5$ & $36 \pm 4$ & $2333 \pm 1044$ & $2410 \pm 837$ \\
M:F & $1: 1$ & & & & \\
\hline
\end{tabular}

PMA, postmenstrual age; BW, birth weight.

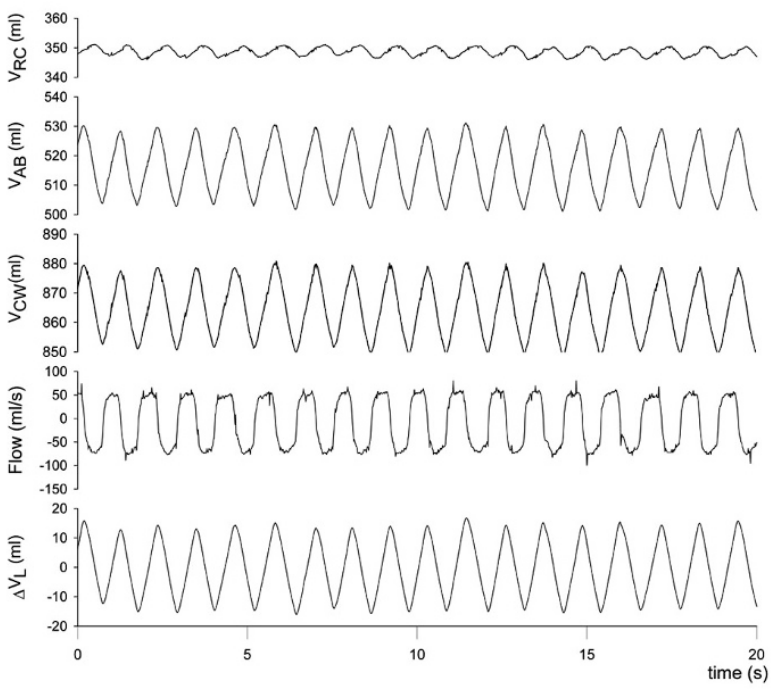

Figure 3. Experimental tracings. $V_{\mathrm{RC}}$, rib cage volume changes; $V_{\mathrm{AB}}$, abdomen volume changes; $V_{\mathrm{CW}}$, total $V_{\mathrm{CW}}$ changes; Flow, airflow measured at the inlet of the full-face mask.

magnitude of volume changes. The correlation analysis on pooled data (Fig. 4) showed a good linear relationship ( $m=$ $0.96, r^{2}=0.95, p<0.001$ ) between $V_{\mathrm{T}, \mathrm{L}}$ and $V_{\mathrm{T}, \mathrm{CW}}$.

The inclusion of body weight and GA as independent variables in the regression analysis did not affect the coefficient of determination, indicating that these two parameters do not introduce bias into the extent of agreement between $V_{\mathrm{T}, \mathrm{L}}$ and $V_{\mathrm{T}, \mathrm{CW}}$.

Table 2 reports $V_{\mathrm{T}, \mathrm{L}}, V_{\mathrm{T}, \mathrm{CW}}$, and the percentage difference between the two for each infant. The average tidal volumes ranged between 12.8 and $31.0 \mathrm{~mL}$, allowing the evaluation of OEP on a wide range of tidal volumes, whereas the average percentage differences ranged between -10.1 and $6.1 \%$.

Compartmental $\mathbf{V}_{C W}$ changes. The comparison between $\%$ diff and $\mathrm{AB} \%$ showed the absence of a trend between the two, suggesting that the geometrical models used to describe the rib cage and of the abdomen are equally accurate. Table 3 shows $V_{\mathrm{T}, \mathrm{CW}}$ measured by OEP and its subdivision into rib cage and abdominal compartments $\left(\mathrm{V}_{\mathrm{T}, \mathrm{RC}}\right.$ and $\mathrm{V}_{\mathrm{T}, \mathrm{AB}}$, respectively), expressed both in absolute values and as a percentage of total $V_{\mathrm{CW}}$ variations. The abdomen contributed $95.3 \pm$ $13.7 \%$ and the rib cage $4.7 \pm 13.7 \%$ to $V_{\mathrm{T}, \mathrm{CW}}$. These values were compared with compartmental $V_{\mathrm{CW}}$ changes reported in the literature for healthy adults in supine position (7). As expected, the main contribution to tidal volume is provided by the abdomen and this contribution is notably higher in infants than in adults.

An example of the Konno-Mead plots obtained in three different representative subjects is shown in Figure 5. In five infants, the rib cage contribution was negative, showing the presence of paradoxical breathing. The phase angle was on average $-61.1 \pm 45.9$, and it was negative for all subjects except one (Table 3), suggesting that the outward motion of the abdomen on average tends to precede ribcage one. 

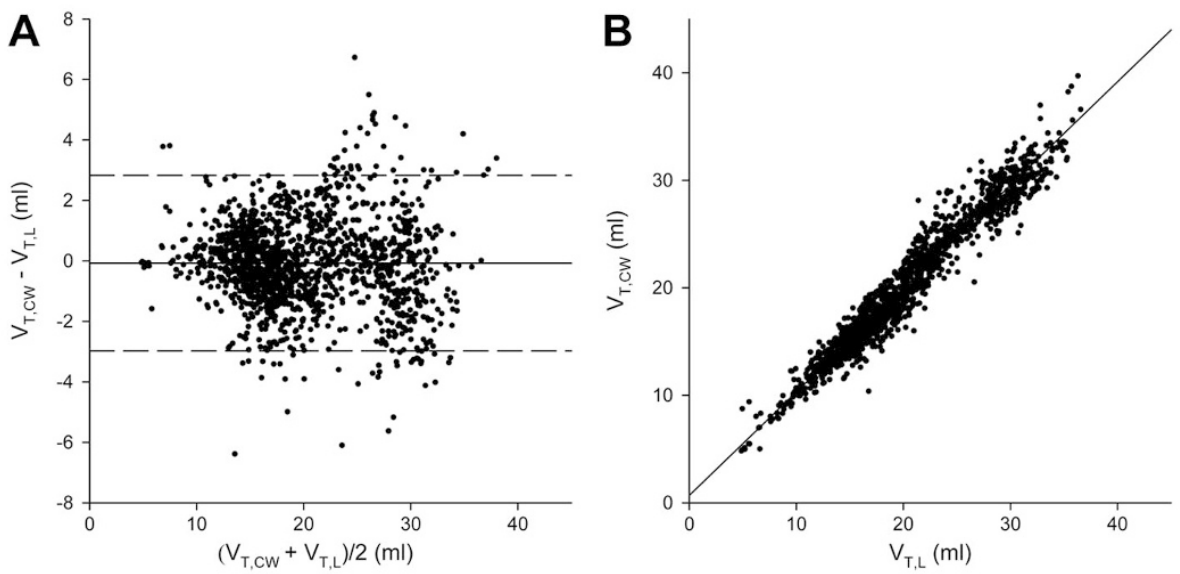

Figure 4. Comparison between pneumotachography and OEP. Left panel: BlandAltman analysis; the solid horizontal line is the mean and the dashed lines are mean \pm 2 SD. Right panel: linear regression between $V_{\mathrm{T}, \mathrm{L}}$ (tidal volume measured by integration of the flow signal) and $V_{\mathrm{T}, \mathrm{CW}}$ (chest wall tidal volume measured by OEP); the slope of the regression line is 0.96 , the intercept is $1 \mathrm{~mL}$, and the coefficient of determination is $r^{2}=0.95$.
Table 2. Comparison between pneumotachography and OEP

\begin{tabular}{lcccr}
\hline Subject \# & $N$ & $V_{\mathrm{TA}}(\mathrm{mL})$ & $V_{\mathrm{T}, \mathrm{CW}}(\mathrm{mL})$ & \multicolumn{1}{c}{$\%$ diff } \\
\hline 1 & 44 & $28.8 \pm 2.2$ & $29.8 \pm 2.3$ & $-3.5 \pm 4.2$ \\
2 & 29 & $29.3 \pm 1.4$ & $28.4 \pm 1.8$ & $2.9 \pm 3.5$ \\
3 & 38 & $31.1 \pm 1.9$ & $29.2 \pm 2.1$ & $6.1 \pm 3.9$ \\
4 & 21 & $19.8 \pm 3.6$ & $19.3 \pm 3.9$ & $2.8 \pm 7.0$ \\
5 & 30 & $22.9 \pm 2.2$ & $22.5 \pm 2.4$ & $1.7 \pm 3.3$ \\
6 & 13 & $30.4 \pm 3.2$ & $29.2 \pm 2.8$ & $3.8 \pm 4.3$ \\
7 & 22 & $14.1 \pm 2.3$ & $14.8 \pm 2.2$ & $-5.7 \pm 8.4$ \\
8 & 28 & $16.7 \pm 1.8$ & $16.1 \pm 1.9$ & $3.8 \pm 8.0$ \\
9 & 10 & $21.2 \pm 2.1$ & $20.2 \pm 1.9$ & $4.4 \pm 2.6$ \\
10 & 24 & $20.2 \pm 2.1$ & $20.3 \pm 2.2$ & $-1.1 \pm 6.3$ \\
11 & 49 & $13.6 \pm 1.4$ & $14.1 \pm 1.5$ & $-3.3 \pm 2.9$ \\
12 & 41 & $22.1 \pm 2.3$ & $24.4 \pm 2.9$ & $-10.1 \pm 5.9$ \\
13 & 35 & $16.4 \pm 4.4$ & $18.0 \pm 4.8$ & $-9.4 \pm 3.9$ \\
14 & 59 & $17.5 \pm 1.4$ & $17.1 \pm 1.2$ & $2.2 \pm 3.6$ \\
15 & 48 & $26.7 \pm 2.9$ & $26.7 \pm 2.6$ & $0.0 \pm 2.6$ \\
16 & 35 & $12.8 \pm 3.6$ & $13.1 \pm 4.0$ & $-1.8 \pm 5.1$ \\
17 & 83 & $14.8 \pm 4.3$ & $14.2 \pm 4.1$ & $3.9 \pm 5.2$ \\
18 & 82 & $15.3 \pm 4.2$ & $15.3 \pm 3.8$ & $-1.2 \pm 8.3$ \\
19 & 60 & $21.0 \pm 3.7$ & $20.7 \pm 4.1$ & $1.1 \pm 8.3$ \\
20 & 20 & $19.0 \pm 2.7$ & $18.3 \pm 2.9$ & $3.1 \pm 12.6$ \\
Mean \pm SD & $39 \pm 20$ & $20.7 \pm 5.9$ & $20.6 \pm 5.6$ & $-0.02 \pm 4.5$ \\
Tot & 771 & & & \\
\hline
\end{tabular}

$N$ indicates the number of analyzed breaths.

\section{DISCUSSION}

The accurate measurement of $\Delta V_{\mathrm{L}}$ is particularly difficult in newborns because they cannot cooperate, and connecting to the airway opening without leaks is extremely difficult. Moreover, the measurement technique has to be noninvasive and should not interfere with the breathing pattern by increasing dead space or breathing load.

Spirometry and PNT are currently used for the measurement of $\Delta V_{\mathrm{L}}$, but they require a connection to the airway opening that may introduce leaks, annoy the infant, and affect the breathing pattern $(1,2)$. Moreover, they cannot measure breath-by-breath changes in absolute lung volumes because of the unavoidable presence of drift in the volume trace, which hinders the assessment of changes in EELV over long timescales.

Infant whole body plethysmography allows the measurement of tidal volumes and EELV, but it requires the use of a cumbersome rigid box, which blocks the access to the infant.
Furthermore, similarly to pneumotachography, it requires a connection to the airway opening and is affected by long-term volume drift.

Respiratory inductive plethysmography (RIP) is a simple and noninvasive respiratory monitoring technique that quantifies breathing pattern, including thoracoabdominal asynchrony, through the changes in the cross-sectional area of the rib cage and the abdominal compartments, without requiring a connection to the airway opening (1). Unfortunately, the calibration procedure is critical and patient-dependent. In addition, measurements are not reliable if the position and condition of the patient differ from those assumed during calibration (3). Moreover, the use of RIP for tracking longterm changes in EELV is still questioned (4,5). Finally, RIP is based on the assumption that the chest wall has only two degrees of freedom (rib cage and abdominal cross-sectional areas). However, the validity of this assumption is questionable, especially with high degrees of distortion and an extremely compliant chest wall, as frequently occurs in neonates (15-17).

OEP has potentially the advantage of overcoming these limitations but, to be applied on newborns, it requires special measurement protocols (i.e., number of markers and their positioning on the chest wall and geometrical models), which have to be developed purposely for this application. In this study, a new experimental protocol designed to apply OEP in newborns was introduced. The accuracy provided by the protocol was evaluated by comparison with pneumotachography during short periods of spontaneous breathing.

Comparison between volume measurements by PNT and OEP. While PNT measures lung gas volume changes, OEP measures the variations of the volume enclosed within the chest wall. For this reason, there are several possible sources of discrepancy between the two measurements.

The measurement of $V_{\mathrm{CW}}$ changes provides an estimation of $\Delta V_{\mathrm{L}}$ in body temperature and pressure, saturated with water vapor (BTPS) conditions. The PNT measures the volume of gas in BTPS during expiration. Conversely, during inspiration, the gas is in ambient temperature and pressure, saturated with water vapor (ATPS) conditions. These differences would require the application of a correction factor of about 8\% (1.091-1.069 for $22-26^{\circ} \mathrm{C}$ ambient temperature in the NICU) to the inspired 
Table 3. Total and compartmental chest wall volume changes

\begin{tabular}{|c|c|c|c|c|c|c|}
\hline Subject & $V_{\mathrm{T}, \mathrm{CW}}(\mathrm{mL})$ & $V_{\mathrm{T}, \mathrm{RC}}(\mathrm{mL})$ & $V_{\mathrm{T}, \mathrm{AB}}(\mathrm{mL})$ & $\mathrm{RC} \%$ & $\mathrm{AB} \%$ & $\theta$ \\
\hline 1 & 29.8 & 5.8 & 24.0 & 19.5 & 80.5 & 5.7 \\
\hline 2 & 28.4 & 0.6 & 27.8 & 2.2 & 97.8 & -68.1 \\
\hline 3 & 29.2 & 8.3 & 20.9 & 28.5 & 71.5 & -7.8 \\
\hline 4 & 19.3 & 1.0 & 18.3 & 5.4 & 94.6 & -21.0 \\
\hline 5 & 22.5 & 1.8 & 20.7 & 7.9 & 92.1 & -45.7 \\
\hline 6 & 29.2 & 5.8 & 23.4 & 19.7 & 80.3 & -16.8 \\
\hline 7 & 14.8 & -4.3 & 19.1 & -29.0 & 129.0 & -141.0 \\
\hline 8 & 16.1 & -0.8 & 16.9 & -4.9 & 104.9 & -119.2 \\
\hline 9 & 20.2 & 0.7 & 19.5 & 3.3 & 96.7 & -75.7 \\
\hline 10 & 20.3 & 3.6 & 16.7 & 17.9 & 82.1 & -19.6 \\
\hline 11 & 14.1 & 2.0 & 12.1 & 14.5 & 85.5 & -35.3 \\
\hline 12 & 24.4 & 2.7 & 21.7 & 11.0 & 89.0 & -20.9 \\
\hline 13 & 18.0 & 0.1 & 17.9 & 0.8 & 99.2 & -56.9 \\
\hline 14 & 17.1 & -1.3 & 18.4 & -7.5 & 107.5 & -90.0 \\
\hline 15 & 26.7 & 2.1 & 24.6 & 7.8 & 92.2 & -66.2 \\
\hline 16 & 13.1 & -2.6 & 15.7 & -20.1 & 120.1 & -157.5 \\
\hline 17 & 14.2 & 1.3 & 12.9 & 9.0 & 91.0 & -58.9 \\
\hline 18 & 15.3 & 0.6 & 14.7 & 3.7 & 96.3 & -78.2 \\
\hline 19 & 20.7 & -1.4 & 22.1 & -6.6 & 106.6 & -119.9 \\
\hline 20 & 18.3 & 2.0 & 16.3 & 11.0 & 89.0 & -29.7 \\
\hline Mean $\pm \mathrm{SD}$ & $20.6 \pm 5.6$ & $1.4 \pm 3.0$ & $19.2 \pm 4.0$ & $4.7 \pm 13.7$ & $95.3 \pm 13.7$ & $-61.1 \pm 45.9$ \\
\hline Adults & 694.0 & 270.0 & 424.0 & 38.9 & 61.1 & NA \\
\hline
\end{tabular}

Adults data are from Ref. 1.
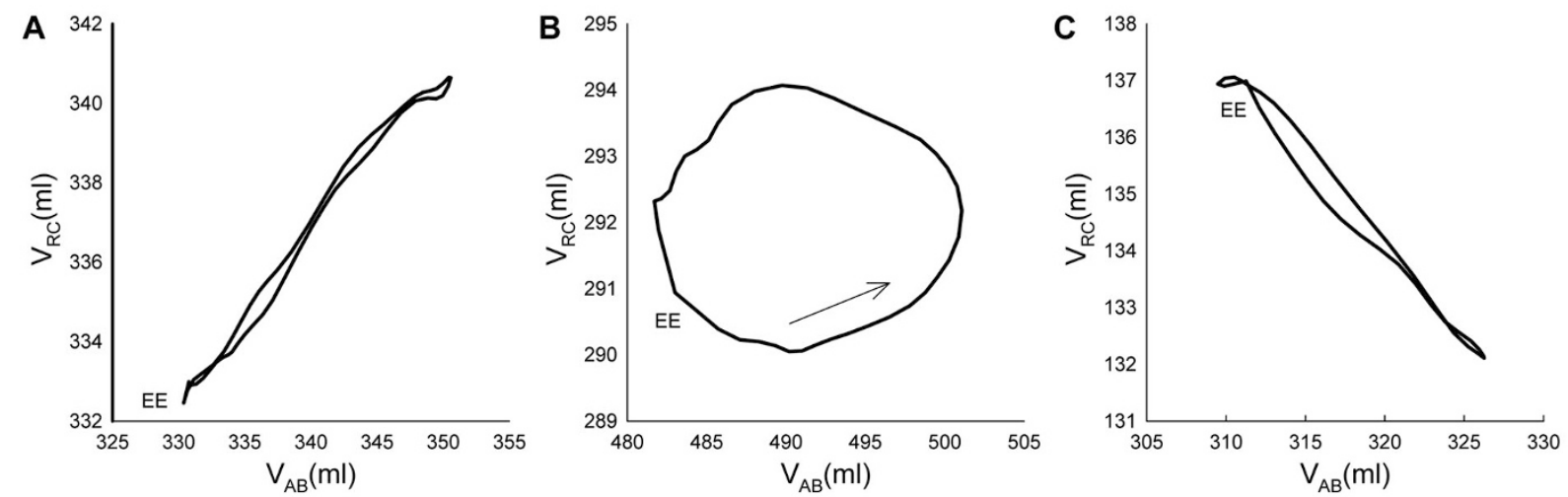

Figure 5. Konno-Mead loops. Konno-Mead loops for three representative subjects presenting synchronous $(A)$, asynchronous $(B)$, and paradoxical $(C)$ chest wall motion.

volume measured by pneumotachography. Because the expected difference is small (or even smaller as the PNT was heated) and is impossible to estimate the real conditions of the gas passing through the PNT mesh (18), it was decided not to correct the data for this source of discrepancy.

Similar considerations can be referred to the different composition of the inhaled and exhaled gas, as the absorption of oxygen and the release of carbon dioxide in the alveolar gas affect the viscosity, leading to possible differences in the pressure drop across the PNT mesh.

Another source of discrepancy between PNT and OEP is represented by possible blood shifts between the trunk and the extremities during breathing, which might produce small but measurable differences between $\Delta V_{\mathrm{L}}$ and changes in $V_{\mathrm{CW}}$, particularly during deep breaths or positive pressure ventilation (6). As in this study, only spontaneous breathing was tested, and it was assumed that changes of gas volume in the lung were equalled to changes in $V_{\mathrm{CW}}$.

Finally, the average \%diff value close to zero suggests that, despite the potential sources of discrepancy described earlier, in our experimental conditions, OEP provides an unbiased, reliable, and accurate estimation of $\Delta V_{\mathrm{L}}$ without requiring any correction factor.

Division of $\mathrm{V}_{C W}$ changes in rib cage and abdominal compartments. Several studies focused on the clinical interest and usefulness of the measurement of thoracoabdominal asynchronies in the diagnosis of sleep disordered breathing or as an indicator of airway obstruction during REM sleep $(19,20)$. Furthermore, it was shown that chest wall motion is strictly correlated with lung mechanics (21) in spontaneously breathing infants with BPD. OEP enables the measurement of volume variations of different compartments, which, in this study, were limited to the rib cage and abdomen. The accuracy of each single compartment was tested by comparing the total accuracy of OEP with the relative contribution of the abdominal compartment, taking advantage of the wide distribution of this parameter in newborns (it ranges from 71.5 to $129 \%$ in the examined patients). In case of a systematically greater error in the volume estimation of one compartment compared with the other, the total accuracy would be biased by the percentage 
contribution of that compartment. The absence of any correlation between these variables indicates that the volume estimation of the two compartments is equally accurate.

Table 3 showed high intersubject variability in the degree of asynchrony between abdominal and rib cage compartments, with paradoxical breathing in five subjects, as expected in healthy infants during spontaneous breathing. Previous studies suggested that the time lag between the movement of rib cage and abdomen differs between term and preterm infants (16), is correlated with changes in the mechanical properties of the lung and chest wall (21-23) and that the percentage rib cage contribution to tidal volume can be used as a measure of rib cage maturation (24). The capability of OEP to provide anatomically based compartmental volumes could help in advancing the research in these applications.

Critiques of the technique. Even if suitable for research, at the actual stage of development, the use of OEP for clinical application in NICU environment is affected by some technical issues, such as the need to attach a high number of markers on the skin of the infant and the need to keep the infant in supine position with the thorax naked. Moreover, movements of the back with respect to the bed may introduce artifacts because the model adopted to close the chest wall surface uses the projection of the initial position of some markers onto the bed plane. Finally, the set-up is cumbersome, the system cannot easily be moved from a bed to another, and the cameras we used cannot see through the incubator.

While we think that the benefits of using OEP fully overcome these limits, future technical improvements are needed to resolve or, at least, reduce the impact of most of these limitations before it is suitable for routine applications at the bedside of clinically ill newborn infants. For instance, markers could be attached to a shirt or tissue strips to simplify their positioning and removal. Recent advances in TV camera technology could also lead to a miniaturization, so that the system could be placed inside the incubator and moved from an incubator to another.

In conclusion, even if further technological developments are welcome, this study demonstrated that OEP could already be used for the accurate assessment of $\Delta V_{\mathrm{L}}$ and its compartmental distribution in term and preterm newborns. Thanks to its specific advantages, it has the potential to be used in many research and clinical applications to further improve the understanding of physiology and pathophysiology of respiration in newborns and at the early stage of development.

Acknowledgments. We thank Daniela Biolo, Alberto Chemotti, Chiara Veneroni, and Francesca Pennati from Politecnico di Milano and Valeria Zimbaldi, Roberta Corbetta, and
Francesca Furlan from Ospedale S. Gerardo for their technical support and assistance during the experimental activity.

\section{REFERENCES}

1. Dolfin T, Duffty P, Wilkes D, England S, Bryan H 1983 Effects of a face mask and pneumotachograph on breathing in sleeping infants. Am Rev Respir Dis 128:977-979

2. Fleming PJ, Levine MR, Goncalves A 1982 Changes in respiratory pattern resulting from the use of a facemask to record respiration in newborn infants. Pediatr Res 16:1031-1034

3. Brown K, Aun C, Jackson E, Mackersie A, Hatch D, Stocks J 1998 Validation of respiratory inductive plethysmography using the Qualitative Diagnostic Calibration method in anaesthetized infants. Eur Respir J 12:935-943

4. Neumann P, Zinserling J, Haase C, Sydow M, Burchardi H 1998 Evaluation of respiratory inductive plethysmography in controlled ventilation: measurement of tidal volume and PEEP-induced changes of end-expiratory lung volume. Chest 113:443-451

5. Werchowski JL, Sanders MH, Costantino JP, Sciurba FC, Rogers RM 1990 Inductance plethysmography measurement of CPAP-induced changes in end-expiratory lung volume. J Appl Physiol 68:1732-1738

6. Aliverti A, Dellaca R, Pelosi P, Chiumello D, Pedotti A, Gattinoni L 2000 Optoelectronic plethysmography in intensive care patients. Am J Respir Crit Care Med 161:1546-1552

7. Aliverti A, Dellaca R, Pelosi P, Chiumello D, Gattinoni L, Pedotti A 2001 Compartmental analysis of breathing in the supine and prone positions by optoelectronic plethysmography. Ann Biomed Eng 29:60-70

8. Cala SJ, Kenyon CM, Ferrigno G, Carnevali P, Aliverti A, Pedotti A, Macklem PT, Rochester DF 1996 Chest wall and lung volume estimation by optical reflectance motion analysis. J Appl Physiol 81:2680-2689

9. Aliverti A, Dellaca RL, Pedotti A 2001 Transfer impedance of the respiratory system by forced oscillation technique and optoelectronic plethysmography. Ann Biomed Eng 29:71-82

10. Dellaca RL, Aliverti A, Pelosi P, Carlesso E, Chiumello D, Pedotti A, Gattinoni L 2001 Estimation of end-expiratory lung volume variations by optoelectronic plethysmography. Crit Care Med 29:1807-1811

11. Ferrigno G, Carnevali P, Aliverti A, Molteni F, Beulcke G, Pedotti A 1994 Three-dimensional optical analysis of chest wall motion. J Appl Physiol 77:12241231

12. Carnevali P, Ferrigno G, Aliverti A, Pedotti A 1996 A new method for 3D optical analysis of chest wall motion. Technol Health Care 4:43-65

13. Bland JM, Altman DG 1986 Statistical methods for assessing agreement between two methods of clinical measurement. Lancet 1:307-310

14. Konno K, Mead J 1967 Measurement of the separate volume changes of rib cage and abdomen during breathing. J Appl Physiol 22:407-422

15. Goldman MD, Pagani M, Trang HT, Praud JP, Sartene R, Gaultier C 1993 Asynchronous chest wall movements during non-rapid eye movement and rapid eye movement sleep in children with bronchopulmonary dysplasia. Am Rev Respir Dis 147:1175-1184

16. Warren RH, Horan SM, Robertson PK 1997 Chest wall motion in preterm infants using respiratory inductive plethysmography. Eur Respir J 10:2295-2300

17. Warren RH, Alderson SH 1986 Breathing patterns in infants utilizing respiratory inductive plethysmography. Chest 89:717-722

18. Gattinoni L, Mascheroni D, Basilico E, Foti G, Pesenti A, Avalli L 1987 Volume/ pressure curve of total respiratory system in paralysed patients: artefacts and correction factors. Intensive Care Med 13:19-25

19. Kohyama J, Sakuma H, Shiiki T, Shimohira M, Hasegawa T 2000 Quantitative analysis of paradoxical inward rib cage movement during sleep in children. Psychiatry Clin Neurosci 54:328-329

20. Kohyama J, Shiiki T, Shimohira M, Hasegawa T 2001 Asynchronous breathing during sleep. Arch Dis Child 84:174-177

21. Allen JL, Greenspan JS, Deoras KS, Keklikian E, Wolfson MR, Shaffer TH 1991 Interaction between chest wall motion and lung mechanics in normal infants and infants with bronchopulmonary dysplasia. Pediatr Pulmonol 11:37-43

22. Locke R, Greenspan JS, Shaffer TH, Rubenstein SD, Wolfson MR 1991 Effect of nasal CPAP on thoracoabdominal motion in neonates with respiratory insufficiency. Pediatr Pulmonol 11:259-264

23. Allen JL, Wolfson MR, McDowell K, Shaffer TH 1990 Thoracoabdominal asynchrony in infants with airflow obstruction. Am Rev Respir Dis 141:337-342

24. Hershenson MB, Colin AA, Wohl ME, Stark AR 1990 Changes in the contribution of the rib cage to tidal breathing during infancy. Am Rev Respir Dis 141:922-925 